\title{
O PERFIL DOS PACIENTES PORTADORES DE COAGULOPATIAS DE UMA REGIÃO DO SUL DO BRASIL
}

Danielle Wisniewski ${ }^{1}$, Ana Cláudia Garabeli Cavalli Kluthcovsky²

RESUMO: Objetivo: analisar o perfil epidemiológico de portadores de coagulopatias cadastrados em um hemonúcleo do Sul do Brasil, em 2006. Metodologia: estudo descritivo, transversal, abordagem quantitativa, que utilizou dados de 39 prontuários. Foram utilizadas freqüências, medidas estatísticas descritivas e valor de p<0,05 para significância estatística. Resultados: a idade média foi 21,1 anos (DP+11,2), 89,8\% do sexo masculino. A maioria procedia dos municípios da região, tinha hemofilia A, não apresentava inibidor, sendo mais freqüente a forma grave da doença. Intercorrências clínicas ocorreram em 25 pacientes (64,1\%), principalmente hemartroses. Intercorrências clínicas apresentaram associação significativa para paciente com diagnóstico de hemofilia A(p=0,01). Durante o ano de 2006, 26 (66,7\%) pacientes receberam infusão de fator, de $5.000 U$ a mais de $40.000 U$ por paciente. Conclusão: este estudo permitiu caracterizar os pacientes com coagulopatias cadastrados e ampliou o conhecimento sobre as patologias descritas, enfatizando a importância do cuidado em Enfermagem.

PALAVRAS-CHAVE: Transtornos da Coagulação Sangüínea; Epidemiologia; Estudos Transversais; Cuidados de Enfermagem.

\section{THE PROFILE OF PATIENTS WITH BLOOD COAGULATION DISORDERS INA REGION IN SOUTHERN BRAZIL}

\begin{abstract}
Objective: to analyze epidemiologic profile in patients with blood coagulation disorders registered in a blood center in the south of Brazil, in 2006. Methodology: descriptive study, cross-sectional, quantitative approach that used data of 39 records. The variables were described by frequencies and descriptive statistical measures. A value of $\mathrm{p}<0.05$ was considered for statistics significance. Results: the average age was 21.1 years (SD+/-11.2), 89.8\% were male. Most of them lived in neighboring municipalities, were type A hemophiliac, with the most severe kind of disease and did not present inhibitor. Twenty-five patients (64.1\%) presented clinical features, mainly hemarthroses. Clinical features presented significant association with the diagnosis of hemophilia A(p=0.01). During 2006, 26 patients (66.7\%) received factor infusion, ranging from 5.000U to more than $40.000 \mathrm{U}$ per patient. Conclusion: this study allowed to characterize the registered patients with blood coagulation disorders and broadened the knowledge on the described pathologies, emphasizing the importance of nursing care.
\end{abstract}

KEYWORDS: Blood Coagulation Disorders, Epidemiology, Cross-Sectional Studies, Nursing Care.

\section{EL PERFIL DE PACIENTES CON COAGULOPATIAS EN UNA REGIÓN EN EL SUR DEL BRASIL}

RESUMEN: Objetivo: analizar los rasgos epidemiológicos de pacientes con coagulopatias registrados en un centro de tratamiento del Sur del Brasil en el ãno de 2006. Metodología: estudio descriptivo, transversal, de abordaje cuantitativa, que utilizó informaciones de 39 prontuarios clínicos. Fueron utilizadas frecuencias, medidas estadísticas descriptivas y valor de $\mathrm{p}<0,05$ para significancia estadística. Resultados: la edad media fue 21,1 años(DP+/-11,2), 89,8\% del sexo masculino, y la mayoría procedía de los municipios de la region y tenía hemofilia A, no apresentaba inhibidor, siendo más frecuente la forma grave de la enfermedad. Intercurrencias clínicas ocurrieron en 25 pacientes (64,1\%), principalmente hemartrosis. Intercurrencias clínicas fueron asociadas con haber diagnóstico de hemofilia A (p=0,01). Durante el año de 2006,26 (el 66,7\%) pacientes recibieron infusión de factor de hasta 5.000U además de 40.000U por paciente. Conclusión: este studio permitió caracterizar los pacientes con coagulopatia cadastrados y amplió el conocimiento acerca de las patologías descriptas, enfatizando el cuidado de enfermería.

PALABRAS CLAVE: Trastornos de coagulación sanguínea; Epidemiologia; Estudios transversales; Cuidados de enfermería.

${ }^{1}$ Estudante do Curso de Graduação em Enfermagem da Universidade Estadual do Centro Oeste-UNICENTRO.

${ }^{2}$ Médica. Mestre em Enfermagem em Saúde Pública. Professora do Departamento de Enfermagem da UNICENTRO.

Autor Correspondente:

Danielle Wisniewski

Rua Luis Cavalcanti da Silva, 182 - 85045-380 - Guarapuava-PR Recebido: 02/11/07

E-mail: danywisny@hotmail.com 


\section{INTRODUÇÃO}

As doenças crônicas são encontradas em qualquer grupo etário, nível socioeconômico e cultura. Enquanto algumas pessoas com problemas crônicos de saúde vivem de forma independente e levam vidas normais com pequenos desconfortos, outras necessitam de monitorização contínua ${ }^{(1)}$. O gerenciamento de todas as condições crônicas é um dos maiores desafios enfrentados pelos sistemas de saúde em todo o mundo ${ }^{(2)}$, e a enfermeira tem um papel fundamental no cuidado a pessoas com doenças crônicas, pois requerem cuidados técnico-científicos permanentes e integrais ${ }^{(3)}$.

Dentre as doenças crônicas, as do sistema hemostático são causas freqüentes de atendimento médico de urgência. Alterações adquiridas ou hereditárias da hemostasia podem se manifestar através de quadros hemorrágicos ou trombóticos ${ }^{(4)}$. Segundo o Ministério da Saúde, havia no Brasil, em 2005, aproximadamente 9.000 portadores de coagulopatias hereditárias $^{(5)}$ e, dessas, as hemofilias e a Doença de von Willebrand (DvW) são as mais comuns ${ }^{(6)}$.

Estima-se que a hemofilia afeta em torno de 400.000 pessoas em todo o mundo ${ }^{(7)}$ e é o distúrbio de sangramento hereditário mais comum, sendo condição recessiva ligada ao sexo (cromossomo X). A incidência é 1 entre 10.000 indivíduos do sexo masculino, sendo 80 a 85\% devidos à hemofilia A e 10 a 15\% à hemofilia $B$. A gravidade da doença varia conforme a deficiência do fator, a hemofilia A é caracterizada por alterações quantitativas ou qualitativas do fator VIII, e a hemofilia $\mathrm{B}$ do fator IX ${ }^{(8)}$.

Devido as hemofilias serem herdadas como traços ligados ao cromossomo $\mathrm{X}$, quase sempre as pessoas afetadas são do sexo masculino, porém as mulheres podem ser portadoras, mas geralmente são assintomáticas. A doença é reconhecida na primeira infância, entretanto, os pacientes com hemofilia leve podem não ser diagnosticados até o início de um trauma sério ou cirurgia ${ }^{(1)}$.

A DvW é um distúrbio do sangramento comum afetando igualmente homens e mulheres, em que ocorre a deficiência do fator de von Willebrand, necessário para a atividade do fator VIII e também para a adesão plaquetária no local da lesão vascular. Mesmo que a síntese do fator VIII seja normal, sua meia-vida é diminuída, apresentando níveis ligeiramente baixos (15 a $50 \%$ do normal) ${ }^{(1)}$. É uma doença hereditária com caráter autossômico dominante, com prevalência de cerca de $1 \%$ da população. No Brasil parece ser subdiagnosticada, pois o número de casos é muito inferior ao dos hemofílicos ${ }^{(6)}$.

A hemorragia é uma das principais características clínicas tanto da hemofilia como da DvW e pode ocorrer espontaneamente ou ser induzida por traumas. O grau, a localização, a duração do dano e a idade do paciente têm impacto sobre a gravidade da hemorragia e sobre as complicações encontradas em ambas as condições. As manifestações clínicas dependem dos sinais e sintomas produzidos pela hemorragia nas várias localidades do corpo, mais comumente na DvW ocorre em tecido muco-cutâneo, e na hemofilia em tecidos mais profundos ${ }^{(9)}$.

Apesar da importância dessas coagulopatias, um estudo realizado em Campinas, São Paulo, demonstrou que, quanto à percepção da comunidade a respeito da hemofilia, muitos não tinham conhecimento sobre essa condição clínica. Entre os doentes estudados, observaram-se altos níveis de auto-rotulação, acompanhados de depressão, ansiedade e insegurança. Os autores também relataram que, por ser a hemofilia um problema limitado a alguns indivíduos e famílias, não colocar em risco a saúde pública, tende a diminuir a sua importância para as autoridades de saúde ${ }^{(10)}$.

As hemofilias são doenças que acarretam alto custo para o Sistema Público de Saúde e ainda maior para os indivíduos acometidos. Além disso, no Brasil as informações sobre a doença são geradas de forma limitada, fragmentada e heterogênea, demonstrado pela pequena quantidade de publicações em língua portuguesa e pelo escasso número de pesquisas aqui realizadas abordando esse assunto ${ }^{(11)}$.

É importante conscientizar-se de que o hemofílico é uma pessoa com as mesmas possibilidades de desenvolvimento físico, intelectual e social de qualquer outra pessoa. Ele possui as mesmas capacidades, aptidões, potencialidades e produtividade, tanto no nível escolar como de trabalho, desde que receba oportunidades para tal. Para que ocorra desenvolvimento normal é necessário um acompanhamento multidisciplinar, envolvendo enfermeiro, hematologista, dentista, fisiatra, fisioterapeuta, psicólogo e infectologista e que o paciente e sua família tenham um conhecimento sobre sua deficiência, pois na medida em que ele for aprendendo, poderá influir nas decisões finais acerca de seu tratamento ${ }^{(12)}$.

Flores et al. ${ }^{(13)}$ demonstraram que os cuidados relacionados ao manuseio do paciente hemofílico foram aprimorados. Como conseqüência deste avanço, 
notaram a importância e a necessidade de que novos conhecimentos, principalmente sobre a terapia de reposição sejam dominados por profissionais da saúde. Nesse contexto, os enfermeiros têm se destacado nos hemocentros e bancos de sangue nas atividades de: planejamento, execução, coordenação, supervisão e avaliação dos procedimentos hemoterápicos e de enfermagem nas unidades, visando a assegurar a qualidade do sangue e hemocomponentes/ hemoderivados coletados e transfundidos ${ }^{(14)}$.

Este estudo justifica-se pela importância de ampliar o conhecimento em base epidemiológica sobre coagulopatias e aprimorar os cuidados necessários no acompanhamento desses pacientes. Estudos epidemiológicos, como fontes de informação de saúde de uma determinada população, investigação de fatores que influenciam a situação de saúde, ou avaliação do impacto de ações para alterar a situação encontrada, fornecem valiosos subsídios para auxiliar tomadas de decisões em todos os níveis da assistência à saúde, tanto no coletivo como no individual ${ }^{(15)}$.

Além do conhecimento da freqüência, da distribuição e dos fatores determinantes das doenças $^{(16)}$, a epidemiologia fornece dados para prevenção, cura e reabilitação, bem como conceitos, raciocínios e técnicas para estudo dos problemas de saúde de populações ${ }^{(15)}$.

A análise do perfil dos pacientes atendidos fornece elementos para subsidiar a implementação de mudanças necessárias na organização do trabalho e conseqüente aprimoramento dos serviços oferecidos. Assim, este estudo objetivou descrever o perfil epidemiológico de pacientes portadores de coagulopatias.

\section{MÉTODOS}

Trata-se de um estudo descritivo, transversal, de abordagem quantitativa, com utilização de dados de prontuários dos pacientes cadastrados e acompanhados em um hemonúcleo do Sul do Brasil, em 2006.

Estudos transversais, apesar das limitações, como impossibilidade de estabelecer a direção temporal das relações encontradas, é um método rápido, simples, de baixo custo e objetivo na coleta de dados ${ }^{(15)}$, sendo um tipo de desenho mais utilizado na epidemiologia atual $^{(17)}$. Além disso, as informações registradas de modo rotineiro, como em prontuários, são muito convenientes para estudos de morbidade, pois se tratam de dados abundantes e de relevo sobre a saúde das pessoas. Apesar das limitações inerentes a essas fontes, como as relativas à seletividade da clientela e à qualidade dos dados, têm sido muito utilizadas ${ }^{(15)}$.

O projeto de pesquisa foi aprovado pelo Comitê de Ética em Pesquisa no dia 06 de dezembro de 2006, sob o número 146/2006, atendendo os princípios éticos estabelecidos pela Resolução 196/96 ${ }^{(18)}$.

Havia 39 pacientes cadastrados na unidade, e todos os prontuários foram analisados. Foram coletadas as informações disponíveis nos prontuários, sobre as seguintes variáveis: idade, sexo, município de origem, diagnóstico, presença de inibidor, gravidade, tratamento, auto-infusão, infusão domiciliar, número e tipo de intercorrências clínicas e quantidade de fator utilizado. A coleta de dados ocorreu de março a junho de 2007.

Após a seleção de dados válidos, os mesmos foram organizados e analisados em planilhas do programa Excel. A descrição das variáveis foi realizada considerando as freqüências absolutas e relativas, e medidas estatísticas descritivas. O teste de KolmogorovSmirnov foi usado para checar normalidade das variáveis. Foram utilizados os testes Exato de Fisher e Qui-quadrado para comparar proporções e o teste $t$ de Student para comparar médias. Um valor de $\mathrm{p}<0,05$ foi considerado para indicar significância estatística.

\section{RESULTADOS E DISCUSSÃO}

Foi realizada a caracterização sociodemográfica e clínica dos pacientes, conforme tabelas 1 e 2 .

Tabela 1 - Distribuição dos pacientes com coagulopatias, segundo características sociodemográficas, 2006. (n=39)

\begin{tabular}{lcc}
\hline Característica & $\mathbf{n}$ & $\mathbf{\%}$ \\
\hline Idade em anos & 6 & 15,4 \\
$6-10$ & 10 & 25,6 \\
$11-20$ & 9 & 23,1 \\
$21-30$ & 6 & 15,4 \\
$31-40$ & 2 & 5,1 \\
41 ou mais & 6 & 15,4 \\
Não informado & & \\
Sexo & 35 & 89,8 \\
Masculino & 4 & 10,2 \\
Feminino & & \\
Município de origem & 13 & 33,3 \\
Município sede & 23 & 59,0 \\
Outros municípios da região & 3 & 7,7 \\
Não informado & & \\
\hline
\end{tabular}


A idade dos pacientes variou de 6 a 44 anos. Os números apresentados na tabela 1 demonstram que a maior parte encontrava-se entre 11 e 30 anos (48,7\%). Aidade média dos pacientes foi de 21,1 anos (DP+11,2).

Em um estudo realizado sobre o cuidado dos pacientes com hemofilia e DvW na cirurgia otorrinolaringológica, observou-se grande variação na idade dos pacientes, de 2 a 62 anos, com idade média de 23,75 anos $^{(12)}$. Em outro estudo sobre o perfil epidemiológico de 113 indivíduos hemofílicos do Estado de Mato Grosso, a idade variou de 0 e 63 anos, com média de 23 anos e a maior concentração ocorreu na faixa etária 5 a 14 anos (32,8\% do total) $)^{(11)}$.

Observa-se dados semelhantes da literatura quanto à idade média dos pacientes, porém diferença quanto à variação das idades, pois o paciente com maior idade neste estudo tinha 44 anos, comparado aos outros dois estudos citados, com maior idade de $62^{(12)}$ e 63 anos $^{(11)}$.

Quanto ao sexo, 89,8\% eram homens e 10,2\% mulheres. O maior percentual de homens acometidos já era esperado, mesmo tendo sido incluídos neste estudo todas as coagulopatias registradas nos prontuários, pois o distúrbio de sangramento hereditário mais comum é a hemofilia ${ }^{(8)}$, sendo condição resultante de herança genética ligada ao cromossomo $\mathrm{X}$ e transmitida quase exclusivamente a indivíduos do sexo masculino(6).

Sobre o município de procedência, a maioria dos pacientes residia nos municípios da região (59\%) e 33,3\% eram residentes no município sede do hemonúcleo, referência para os 19 municípios da região.

O indivíduo hemofílico pode necessitar, a qualquer momento, da reposição urgente do fator de coagulação, terapêutica de alto custo e geralmente disponível em poucos centros especializados. Esse fato cria uma situação psicossocial peculiar para esse indivíduo: a de alguém que vive sob a constante ameaça de um sangramento inesperado e na dependência, em termos de distância geográfica e disponibilidade, de um centro especializado de tratamento. Assim, pacientes que residem em localidades mais próximas podem ser assistidos em seus episódios hemorrágicos sem perda de tempo, e os demais podem sofrer sérios danos, inclusive risco de vida, por não terem atendimento específico e sistematizado em seus municípios de origem ${ }^{(11)}$.

Tabela 2 - Distribuição dos pacientes com coagulopatias, segundo características clínicas, 2006. (n=39)

\begin{tabular}{lcc}
\hline Característica & n & \% \\
\hline Diagnóstico & 30 & 76,9 \\
Hemofilia A & 6 & 15,4 \\
Doença de von Willebrand & 2 & 5,1 \\
Hemofilia B & 1 & 2,6 \\
Doença de von Willebrand ou Hemofilia A* & & \\
Presença de inibidor & 32 & 82,1 \\
Não & 5 & 12,8 \\
Sim & 2 & 5,1 \\
Não informado & & \\
Gravidade da doença & 15 & 38,5 \\
Grave & 13 & 33,3 \\
Moderada & 4 & 10,2 \\
Leve & 7 & 18,0 \\
Não informado & & \\
Tratamento & 25 & 64,1 \\
FVIII & 7 & 18,0 \\
F8Y & 5 & 12,8 \\
Feiba e FVIII & 2 & 5,1 \\
FIX & & \\
Auto-infusão & 37 & 94,9 \\
Não & 2 & 5,1 \\
Sim & & \\
Infusão domiciliar & 20 & 51,3 \\
Não & 19 & 48,7 \\
Sim & & \\
\hline & & \\
\hline
\end{tabular}

*Paciente com diagnóstico não definido

A hemofilia A foi o diagnóstico mais freqüente e representou $76,9 \%$ dos pacientes cadastrados, Tabela 2. Considerando-se apenas os pacientes com hemofilia A (30 casos) e com hemofilia B ( 2 casos), houve 93,7\% dos casos de hemofilia A e 6,3\% de hemofilia B, uma taxa maior de casos de $\mathrm{A}$ e menor de $\mathrm{B}$, quando se compara com dados de literatura, com relatos de 80 a $85 \%$ de hemofília A e 10 a $20 \%$ hemofilia $\mathrm{B}^{(7)}$.

Todos os casos de hemofilia A eram do sexo masculino. Dos 6 casos $(15,4 \%)$ de DvW, 2 eram do sexo masculino e 4 do sexo feminino. Os dois casos (5,1\%) de hemofilia B e um caso (2,6\%) com diagnóstico não definido entre DvW e hemofilia A eram homens.

Dentre os 39 prontuários analisados, em 5 $(12,8 \%)$ deles havia relato de presença de inibidor. O desenvolvimento de inibidores é uma das complicações 
do tratamento, e são anticorpos da classe IgG dirigidos contra o fator VIII ou IX. Os pacientes mais afetados são, geralmente, aqueles acometidos por hemofilia grave, manifestando má resposta ao tratamento habitual ou aumento da freqüência e/ou gravidade dos sangramentos ${ }^{(6)}$. A incidência do desenvolvimento de inibidores é cerca de 5-15\% dos pacientes com hemofilia A e cerca de $3 \%$ nos pacientes com hemofilia $\mathrm{B}^{(8)}$.

Quanto à gravidade, observou-se neste estudo que a maior parte dos casos era grave (38,5\%), seguido de $33,3 \%$ de casos moderados e 10,2\% leves. Esta classificação baseia-se na atividade residual dos fatores VIII ou IX, ou seja, a forma grave caracteriza-se pela atividade do fator menor que $1 \%$, a moderada por atividade do fator de 1 a $5 \%$, e a leve por atividade do fator de 5 a $40 \%^{(7)}$.

O tratamento mais utilizado foi o fator VIII $(64,1 \%)$, pois corresponde ao tratamento da coagulopatia mais freqüente, a hemofilia $\mathrm{A}$. $\mathrm{O}$ fator F8Y (concentrado de fator VIII de pureza intermediária 8Y) foi utilizado naqueles pacientes portadores de DvW, feita nos pacientes que possuem inibidor e FVIII nos pacientes com hemofilia A.

Observa-se que a infusão domiciliar foi realizada por $48,7 \%$ dos pacientes e somente $5,1 \%$ dos pacientes realizavam a autoinfusão, ambos necessitando treinamento prévio realizado pela enfermeira da unidade.

Para participar destes programas, o paciente é avaliado de acordo com alguns critérios, como: ter mais de três anos de idade; ter bom acesso venoso periférico; ter bons conhecimentos sobre hemofilia; querer ser treinado para conseguir alcançar um bom nível de entendimento e estar tecnicamente bem treinado $^{(19)}$.

Destaca-se a importância da terapia domiciliar, que obteve grande aceitação pelos pacientes com hemofilia e o reconhecimento da Federação Mundial de Hemofilia com a ressalva de que o programa seria um integrante adicional ao tratamento multidisciplinar do paciente com hemofilia ${ }^{(6)}$.

Sobre as intercorrências clínicas ocorridas em 2006, 35,9\% dos pacientes não apresentaram, enquanto 64,1\% apresentaram (Tabela 3), variando de 1 até mais de 41 por paciente no ano, em um total de 460 .

A maioria (48,0\%) dos pacientes apresentou de 1 a 10 intercorrências. Três pacientes apresentaram 41 ou mais no ano, o que daria uma média aproximada de 3 intercorrências por mês/paciente, demonstrando a morbidade da doença nestes casos.
Tabela 3 - Distribuição dos pacientes com coagulopatias, segundo presença, tipo e quantidade por faixa etária de intercorrências clínicas, 2006

\section{Paracterística}

Com intercorrência 2564,1

Sem intercorrência

$14 \quad 35,9$

Tipo de intercorrência $(n=460)$

Hemartrose de cotovelo

$127 \quad 27,6$

Hemartrose de tornozelo

$\begin{array}{ll}78 & 17,0\end{array}$

Hemartrose de joelho

$\begin{array}{ll}73 & 15,9\end{array}$

Hemartrose de ombro

$26 \quad 5,7$

Sangramento gengival

224,8

Hemartrose em punho

194,1

Hematoma em pé

$12 \quad 2,6$

Epistaxe

$11 \quad 2,4$

Hematoma em coxa

$10 \quad 2,2$

Hematoma em panturrilha

$10 \quad 2,2$

Hematoma braço

$10 \quad 2,2$

Hematoma perna

$9 \quad 1,9$

Hematoma lombar

Hematúria

61,3

30,6

Outras

$44 \quad 9,5$

Número de intercorrências no ano $(n=460)$, por faixa etária dos pacientes (em anos)

1 a 5 $0 \quad 0$

6 a 10 $40 \quad 8,7$

11 a 15 $108 \quad 23,5$

16 a 20

$\begin{array}{ll}60 & 13,0\end{array}$

21 a 25

$104 \quad 22,6$

26 a 30

$5 \quad 1,1$

31 a 35

$12 \quad 2,6$

Maior de 36

$97 \quad 21,1$

Idade não informada

$34 \quad 7,4$

As intercorrências clínicas que mais ocorreram foram as hemartroses somando $70,3 \%$, dado este semelhante à literatura, que cita ocorrência de hemartroses entre 70 a $80 \%{ }^{(7)}$ e em cerca de $75 \%$ dos casos de sangramentos ${ }^{(1)}$. Dentre todas, a intercorrência mais freqüente foi a hemartrose de cotovelo $(27,6 \%)$. 
Considerando-se apenas as hemartroses, a articulação acometida com maior freqüência foi a de cotovelo (39,3\%), seguida de tornozelo (24,1\%), joelho (22,6\%), ombro (8,1\%) e punho (5,9\%). Esses dados diferem da literatura, que relata maior freqüência de hemartrose nas articulações do joelho (45\%), cotovelo (30\%), tornozelo (15\%), ombro (3\%), punho (3\%), quadril $(2 \%)$ e outras $(2 \%)^{(7)}$.

Os pacientes geralmente notam dor na articulação antes de estarem atentos ao edema e à limitação dos movimentos. Hemorragias articulares recorrentes podem resultar em danos graves, como dor crônica ou anquilose da articulação. Muitos pacientes com grave deficiência do fator ficam com deformidades ${ }^{(1)}$.

Estudo realizado em São Paulo por Galante et al. ${ }^{(20)}$, com 62 crianças hemofílicas com e sem sinovite crônica, de 3 a 7 anos, e 20 crianças constituíram o grupo controle, com o objetivo de avaliar o alinhamento postural dos membros inferiores e o desempenho funcional de crianças hemofílicas, relacionando-os com a incidência de hemartroses. Os autores concluíram que a média de sangramentos dos pacientes hemofílicos aumentou com a idade, e pareceu estar relacionada com o grau de comprometimento articular. As crianças hemofílicas de 7 anos apresentaram menor desempenho funcional quando comparadas às outras crianças.

Neste estudo, observou-se aumento progressivo do número de intercorrências entre 1 e 15 anos, sendo que entre 11 e 15 anos ocorreu o maior número, entre todas as faixas etárias (23,5\%). Importante ressaltar que nesta distribuição foram consideradas todas as intercorrências, não apenas os casos de hemartroses.

Além dos sangramentos articulares, podem ocorrer sangramentos em músculos/partes moles, outros tipos de sangramento e sangramento do Sistema Nervoso Central $^{(7)}$. O local considerado de maior risco é a cabeça (sangramento intracraniano ou

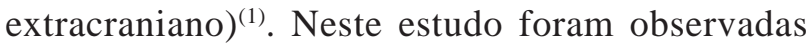
intercorrências de sangramento gengival $(4,7 \%)$, hematoma em pé (2,6\%), epistaxe (2,4\%), hematoma em coxa $(2,2 \%)$, panturrilha $(2,2 \%)$ braço $(2,2 \%)$ e perna $(2,2 \%)$, entre outras menos freqüentes.

Outras complicações têm sido descritas, como a presença de candidíase na saliva de pacientes hemofílicos. O estudo sugeriu que pacientes hemofílicos albergavam mais freqëntemente a Cândida na cavidade bucal e em maiores quantidades que indivíduos do grupo controle, independentemente de infecção viral, próteses dentárias, transfusões de hemoderivados e fluxo salivar $^{(21)}$. Neste estudo, nenhum caso foi relatado de candidíase na cavidade bucal. Também são relatados como complicações crônicas da hemofilia a artropatia hemofílica crônica (sinovite crônica, deformidade articular), as contraturas, o pseudotumor (tecidos moles e ossos), as fraturas, a formação de inibidores contra fatores VIII/IX, as infecções transfusionais: vírus da imunodeficiência humana (HIV), vírus da hepatite B (HBV), vírus da hepatite C (HCV), vírus da hepatite A, Parvovírus B19 e outros ${ }^{(7)}$.

A Tabela 4 apresenta a associação entre variáveis sociodemográficas e clínicas de pacientes com coagulopatias, que apresentaram ou não intercorrências clínicas durante o ano de 2006.

Foram comparados os grupos de pacientes que apresentaram intercorrências clínicas durante 2006 com os que não apresentaram, em relação às variáveis: idade, sexo, diagnóstico, presença de inibidor e gravidade da doença. A única variável que apresentou diferença estatisticamente significativa entre os grupos foi o diagnóstico da coagulopatia ( $\mathrm{p}=0,01)$, com associação entre ter diagnóstico de hemofilia A e a presença de intercorrências. Assim, os resultados sugerem que os pacientes que tem diagnóstico de hemofilia A deveriam receber especial atenção quanto à possibilidade de intercorrências clínicas.

Na Tabela 5 está a distribuição dos pacientes com coagulopatias, segundo infusão e quantidade de fator utilizado em 2006. Quanto à infusão e quantidade de fator utilizado pelos pacientes em 2006, as unidades recebidas por paciente variaram de até $5.000 \mathrm{U}$ a mais de $40.000 \mathrm{U}$, sendo que $19,2 \%$ dos pacientes receberam mais de 40.000 unidades. Um paciente utilizou 97.000U no ano de 2006, com uma média de 8.000U por mês. Além disso, 6 pacientes (23,1\%) necessitaram receber infusão de fator pelo menos uma vez por mês, durante todo o ano de 2006.

Para o tratamento das hemofilias, utiliza-se a administração dos fatores de coagulação VIII ou IX, dependendo do tipo de coagulopatia, de preferência liofilizados, logo após um sangramento ou antes de uma cirurgia. Infelizmente, é muito difícil manter níveis constantes de fatores VIII ou IX nos hemofílicos por administração contínua, seja pela curta vida média dos fatores (8 a 12 horas no fator VIII, e 24 horas no fator IX), ou pelo alto preço ${ }^{(10)}$.

Apesar disso, a expectativa de vida de pessoas que nascem com hemofilia e que têm acesso a tratamento adequado, aproxima-se da dos indivíduos normais $^{(7)}$. Contudo, fato importante foi observado ao se comparar por faixa etária a população brasileira e 
a população de pacientes com deficiência da coagulação em termos percentuais, que demonstrou uma expectativa de vida menor para os pacientes coagulopatas $^{(22)}$. Assim, enfatiza-se a importância do atendimento integral ao paciente e disponibilização de tratamento adequado.

Tabela 4 - Associação entre variáveis (sociodemográficas e clínicas) de pacientes com coagulopatias que apresentaram ou não intercorrências clínicas em 2006. (n=39)

\begin{tabular}{lccccc}
\hline Variáveis & $\begin{array}{c}\text { com intercorrência } \\
\mathrm{n}=25\end{array}$ & $\%$ & $\begin{array}{c}\text { sem intercorrência } \\
\mathrm{n}=14\end{array}$ & $\%$ & $\mathrm{p}$ \\
\hline Idade em anos & & & & & \\
Média & 20,3 & & & & \\
Sexo & & & & & \\
Feminino & 1 & 25,0 & 3 & 75,0 & \\
Masculino & 24 & 68,6 & 11 & 31,4 & $0,12^{* *}$ \\
Diagnóstico & & & & & \\
Hemofilia A & 23 & 76,7 & 7 & 23,3 & \\
Hemofilia B & 1 & 50,0 & 1 & 50,0 & \\
Doença de von Willebrand & 1 & 16,7 & 5 & 83,3 & \\
Hemofilia A e Doença de von Willebrand & 0 & 0 & 1 & 100,0 & $0,01^{* * *}$ \\
Presença de inibidor & & & & & \\
Não & 21 & 65,6 & 11 & 34,4 & \\
Sim & 4 & 80,0 & 1 & 20,0 & $0,65^{* *}$ \\
Gravidade da doença & & & & & \\
Leve & 2 & 50,0 & 2 & 50,0 & \\
Moderada & 11 & 84,6 & 2 & 15,4 & \\
Grave & 9 & 64,3 & 5 & 35,7 & $0,31^{* * * *}$ \\
\hline
\end{tabular}

Consideradas apenas as informações válidas. * Teste $t$ de Student; **Teste Exato de Fisher; *** Teste Qui-quadrado

Tabela 5 - Distribuição dos pacientes com coagulopatias, segundo infusão e quantidade de fator utilizado em 2006. $(\mathrm{n}=39)$

\begin{tabular}{lcc}
\hline Característica & N & \% \\
\hline Receberam infusão (n=39) & & \\
Sim & 26 & 66,7 \\
Não & 13 & 33,3 \\
Número de unidades recebidas, em 2006 & & \\
(n=26) & 5 & 19,2 \\
Até 5.000U & 2 & 7,7 \\
De 5.001 a 10.000U & 5 & 19,2 \\
De 10.001 a 15.000U & 2 & 7,7 \\
De 15.001 a 20.000U & 4 & 15,5 \\
De 20.001 a 25.000U & 1 & 3,8 \\
De 25.001 a 30.000U & 2 & 7,7 \\
De 30.001 a 40.000U & 5 & 19,2 \\
mais de 40.000U & &
\end{tabular}

\section{CONSIDERAÇÕES FINAIS}

Apesar das limitações deste estudo, como a utilização de prontuários para a coleta de dados com alguns dados incompletos e o pequeno número de pacientes estudados, acredita-se que este estudo atingiu seus objetivos e ampliou o conhecimento quanto ao perfil dos pacientes com coagulopatias cadastrados em um hemonúcleo no Sul do Brasil, e sobre as patologias descritas.

Destaca-se a importância da atenção à saúde que fornece informação oportuna, apoio e monitoramento constante, o que pode reduzir a carga dessas doenças crônicas aos pacientes, à população, aos sitemas de saúde aos custos em saúde.

Propõe-se a elaboração de outros estudos relacionados às coagulopatias, com o intuito de contribuir para a identificação das necessidades de cuidados desta clientela pelos profissionais de saúde, especialmente o 
enfermeiro, bem como maior divulgação dessas doenças para a população em geral, com conseqüente melhoria na qualidade de vida dos pacientes.

\section{REFERÊNCIAS}

1. Smeltzer SC, Bare BG. Tratado de enfermagem médicocirúrgica. Rio de Janeiro: Guanabara Koogan; 2002.

2. Organização Mundial da Saúde. Cuidados inovadores para as condições crônicas: componentes estruturais de ação: relatório mundial. Brasília; 2003.

3. Balduino AFA, Labronici LM, Maftum MA, Mantovani MF, Lacerda MR. Um marco de referência para a prática da Enfermeira a pacientes com doenças crônicas à luz de Wanda de Aguiar Horta. Cogitare Enferm. 2007 Jan/ Mar;12(1):89-94.

4. Pintão MCT, Garcia AA. Tratamento de distúrbios hemostáticos em urgência médica. Medicina. 2003 Abr/ Dez;36:439-45.

5. Ministério da Saúde (BR). Saúde, Brasil. Hemofilia. Brasília: Ministério da Saúde; 2005.

6. Ministério da Saúde (BR). Manual de tratamento das coagulopatias hereditárias. Brasília: Ministério da Saúde; 2005.

7. World Federation of Hemophilia. WFH Treatment Guidelines Working Group. Guidelines for the Management of Hemophilia. Quebec: World Federation of Hemophilia; 2005.

8. Secretária de Estado da Saúde (SESA). Normas para atendimento aos hemofílicos. Curitiba: Centro de Hematologia e Hemoterapia do Paraná(HEMEPAR); 1996.

9. Boayue KB, Bell BA. Sangramento na hemofilia e doença de von Willebrand - características e conduta. Int J Pediatr Hematol Oncol. 1994;1:449-61.

10. Caio VM, Silva RBP, Magna LA, Ramalho AS. Genética comunitária e hemofilia em uma população brasileira. Cad Saúde Públ. 2001 Mai/Jun;17(3):595-605.

11. Carapeba RAP. Características epidemiológicas dos portadores de hemofilia no Estado de Mato Grosso [dissertação]. Cuiabá: Universidade Federal de Mato Grosso; 2006 [acesso em 2008 Mai 27]. Disponível em: www.hemofiliabrasil.org.br

12. Marques MPC, Leite EST. Cuidados nos pacientes com hemofilia e doença de von Willebrand na cirurgia eletiva otorrinolaringológica. Rev Bras Otorrinolaringol. 2003 Jan/Fev;69(1):40-6.

13. Flores RPG, Bagatini A, Santos ATL, Gomes CR, Fernandes MS, Molon RP. Hemofilia e anestesia. Rev Bras Anestesiol. 2004 Nov/Dez;54(6):865-71.

14. Benetti SRMD, Lenardt MH, Tuoto FS. As transfusões sanguíneas: o sangue e o sistema de conhecimento das pessoas. Cogitare Enferm. 2003 Jul/Dez;8(2):54-60.

15. Pereira MG. Epidemiologia: teoria e prática. Rio de Janeiro: Guanabara Koogan, 1995.

16. Hennekens CH, Buring G. Epidemiology in medicine. Boston: Little Brown, 1987. Definition and background; p.3-15.

17. Almeida Filho N, Rouquaryol MZ. Introdução à epidemiologia moderna. Belo Horizonte/ Salvador/Rio de Janeiro: COOPMED/APCE/ABRASCO, 1992. Metodologia da pesquisa epidemiológica; p. 79-101.

18. Ministério da Saúde (BR). Conselho Nacional de Saúde. Diretrizes e normas regulamentadoras de pesquisa envolvendo seres humanos. Resolução $n^{\circ}$ 196, 10 de Outubro de 1996. Brasília; 1996.

19. Sandoval E, coordenadora. Auto-infusão e infusão domiciliar. São Paulo: Centro de Hemofilia Louis Aledort da Fundação Pró-Sangramento/ Hemocentro de São Paulo - Novo Care/ Novo Nordisk Services. 20 p. [Acesso em: 2006 Out 20]. Disponível em: www.novocare.com.br/ folhetos/livreto_hemofilia_02.pdf.

20. Galante GA, Azevedo CSA, Mello M, Tanaka C, D’Amico EA. Avaliação do alinhamento postural e do desempenho em atividades funcionais de crianças hemofílicas em idade inferior a sete anos, com e sem sinovite crônica: correlação com a incidência de hemartroses. Rev Bras Fisioter. 2006 Fev;10(2):171-6.

21. Pereira CM, Pires FR, Corrêa MEP, Júnior OH, Almeida OP. Cândida na saliva de pacientes hemofílicos brasileiros. J Appl Oral Sci. 2004 Out/Dez;12(4):301-6.

22. Ministério da Saúde (BR). Agência Nacional de Vigilância Sanitária (ANVISA). Relatório estatístico do cadastro de coagulopatias hereditárias. Brasília; 2002. 